ESAIM: PROCEEDINGS, April 2009, Vol. 26, p. 94-99

H. Ammari, Editor

\title{
ON STABLE DETERMINATION OF POTENTIAL BY BOUNDARY MEASUREMENTS
}

\author{
R.G. NovikOV ${ }^{1}$ AND N.N. NovikOva ${ }^{2}$
}

Abstract. We give new stability estimates for the Gel'fand-Calderon inverse boundary value problem.

\section{INTRODUCTION}

Consider the equation

$$
-\Delta \psi+v(x) \psi=0, \quad x \in D,
$$

where

$$
D \text { is an open bounded domain in } \mathbb{R}^{d}, \partial D \in C^{2}, \quad v \in L^{\infty}(D), \quad d \geq 2 .
$$

We assume also that

$$
0 \text { is not a Dirichlet eigenvalue forthe operator }-\Delta+v \text { in } D \text {. }
$$

Equation (1.1) arises, in particular, in quantum mechanics, acoustics, electrodynamics. Formally, (1.1) looks as the Schrödinger equation with potential $v$ at zero energy.

Consider the map $\Phi$ such that

$$
\left.\frac{\partial \psi}{\partial \nu}\right|_{\partial D}=\Phi\left(\left.\psi\right|_{\partial D}\right)
$$

for all sufficiently regular solutions $\psi$ of $(1.1)$ in $\bar{D}=D \cup \partial D$, where $\nu$ is the outward normal to $\partial D$. The map $\Phi$ is called the Dirichlet-to-Neumann map for equation (1.1) and is considered as boundary measurements for (1.1).

We consider the following inverse boundary value problem for equation (1.1):

Problem 1.1. Given $\Phi$, find $v$.

This problem can be considered as the Gel'fand inverse boundary value problem for the Schrödinger equation at zero energy (see [8], [14]). This problem can be also considered as a generalization of the Calderon problem of the electrical impedance tomography (see [5], [19], [14]). Concerning results given in the literature on Problem 1.1 (in its Calderon or Gel'fand form ) see [10], [19], [9] (note added in proof), [14], [1], [12], [13], [4], [18], [11], [15], [17], [2] and references therein.

In the present article we show that the Alessandrini stability estimates of [1] for Problem 1.1 in dimension $d \geq 3$ (see Theorem 2.1 of the next section) admit some principle improvement. Our new stability estimates (see Theorem 2.2 of the next section) are obtained by methods developed in [15], [16], [17]. These methods

\footnotetext{
${ }^{1}$ CNRS, Laboratoire de Mathématiques Jean Leray (UMR 6629), Université de Nantes, BP 92208, F-44322, Nantes cedex 03,

France; e-mail: novikov@math.univ-nantes.fr

2 MITP AN, Profsoyuznaya 84/32, 117997, GSP-7, Moscow, Russia.
}

(c) EDP Sciences, SMAI 2009 
include, in particular: (1) the $\bar{\partial}$ - approach to inverse "scattering" at zero energy in dimension $d \geq 3$, going back to [3], [9], and (2) the reduction of Problem 1.1 to inverse "scattering" at zero energy, going back to [14].

The present article is organized as follows. In Section 2, we formulate and discuss old and new stability estimate for Problem 1.1. In Section 3, we recall (a) definition and some properties of the Faddeev functions and (b) formulation of the inverse "scattering" problem for the Schrödinger equation at zero energy (Problem 3.1). In Section 4, we recall formulas and equations of [14], [15] reducing Problem 1.1 to Problem 3.1. In Section 5 , we recall an approximate reconstruction method of [14] for Problem 1.1. In Section 6 we prove Theorem 2.2 in the Born approximation.

\section{Stability EStimates}

We assume for simplicity that

$D$ is an open bounded domain in $\mathbb{R}^{d}, \partial D \in C^{2}, v \in W^{m, 1}\left(\mathbb{R}^{d}\right)$ for some $m>d$, supp $v \subset D, d \geq 2$,

where

$$
W^{m, 1}\left(\mathbb{R}^{d}\right)=\left\{v: \partial^{J} v \in L^{1}\left(\mathbb{R}^{d}\right),|J| \leq m\right\}, \quad m \in \mathbb{N} \cup 0
$$

where

$$
J \in(\mathbb{N} \cup 0)^{d}, \quad|J|=\sum_{i=1}^{d} J_{i}, \quad \partial^{J} v(x)=\frac{\partial^{|J|} v(x)}{\partial x_{1}^{J_{1}} \ldots \partial x_{d}^{J_{d}}}
$$

Let

$$
\|v\|_{m, 1}=\max _{|J| \leq m}\left\|\partial^{J} v\right\|_{L^{1}\left(\mathbb{R}^{d}\right)}
$$

Let

$$
\|A\| \text { denote the norm of an operator } A: L^{\infty}(\partial D) \rightarrow L^{\infty}(\partial D) .
$$

We recall that if $v_{1}, v_{2}$ are potentials satisfying $(1.2),(1.3)$, where $D$ is fixed, then

$$
\Phi_{1}-\Phi_{2} \text { is a compact operator in } L^{\infty}(\partial D)
$$

where $\Phi_{1}, \Phi_{2}$ are the DtN maps for $v_{1}, v_{2}$ respectively, see [14], [15]. Note also that $(2.1) \Rightarrow(1.2)$.

Theorem 2.1 (variation of the result of [1]). Let conditions (1.3), (2.1) hold for potentials $v_{1}$ and $v_{2}$, where $D$ is fixed, $d \geq 3$. Let $\left\|v_{j}\right\|_{m, 1} \leq R, j=1,2$, for some $R>0$. Let $\Phi_{1}, \Phi_{2}$ denote the DtN maps for $v_{1}$, $v_{2}$, respectively. Then

$$
\left\|v_{1}-v_{2}\right\|_{L^{\infty}(D)} \leq C_{1}\left(\ln \left(1+\left\|\Phi_{1}-\Phi_{2}\right\|^{-1}\right)\right)^{-\alpha_{1}},
$$

where $C_{1}=C_{1}(R, D, m), \alpha_{1}=(m-d) / m,\left\|\Phi_{1}-\Phi_{2}\right\|$ is defined according to (2.4).

Theorem 2.1 follows from formulas (3.9)-(3.11), (4.1) (of Sections 3 and 4).

A disadvantage of estimate $(2.6)$ is that

$$
\alpha_{1}<1 \text { for any } m>d \text { even if } m \text { is very great. }
$$

Theorem 2.2. Let the assumptions of Theorem 2.1 hold. Then

$$
\left\|v_{1}-v_{2}\right\|_{L^{\infty}(D)} \leq C_{2}\left(\ln \left(1+\left\|\Phi_{1}-\Phi_{2}\right\|^{-1}\right)\right)^{-\alpha_{2}}
$$

where $C_{2}=C_{2}(R, D, m), \alpha_{2}=m-d,\left\|\Phi_{1}-\Phi_{2}\right\|$ is defined according to (2.4).

A principal advantage of estimate (2.8) in comparison with (2.6) is that

$$
\alpha_{2} \rightarrow+\infty \text { as } m \rightarrow+\infty
$$


in contrast with (2.7).

In the Born approximation, that is in the linear approximation near zero potential, Theorem 2.2 is proved in Section 6.

For sufficiently small $R$ in dimension $d=3$, Theorem 2.2 follows from (3.9) (of Section 3) and results of [15], [17]. The scheme of our proof for this case is, actually, similar to the scheme of our proof for the case of the Born approximation. The main difference is that instead of the inverse Fourier transform (used in Section 6) we use now the zero-energy inverse "backscattering" transform of [17]. We plan to give this "nonlinear" "small-norm" proof in a separate article.

In the general case, the proof of Theorem 2.2 is not completed yet. However, except restrictions in time, we see no difficulties for completing this proof by methods of [15], [16], [17].

\section{FADDEEV FUnCTIONS}

We consider the Faddeev functions $G, \psi$ and $h$ (see [6], [7], [9], [15]):

$$
\begin{gathered}
G(x, k)=e^{i k x} g(x, k), \quad g(x, k)=-\left(\frac{1}{2 \pi}\right)^{d} \int_{\mathbb{R}^{d}} \frac{e^{i \xi x} d \xi}{\xi^{2}+2 k \xi}, \\
\psi(x, k)=e^{i k x}+\int_{\mathbb{R}^{d}} G(x-y, k) v(y) \psi(y, k) d y,
\end{gathered}
$$

where $x \in \mathbb{R}^{d}, k \in \Sigma$,

$$
\begin{aligned}
& \Sigma=\left\{k \in \mathbb{C}^{d}, \quad k^{2}=k_{1}^{2}+\ldots+k_{d}^{2}=0\right\} ; \\
& h(k, l)=\left(\frac{1}{2 \pi}\right)^{d} \int_{\mathbb{R}^{d}} e^{-i l x} v(x) \psi(x, k) d x,
\end{aligned}
$$

where $(k, l) \in \Theta$,

$$
\Theta=\{k \in \Sigma, \quad l \in \Sigma: \operatorname{Im} k=\operatorname{Im} l\} .
$$

We recall that:

$$
\Delta G(x, k)=\delta(x), \quad x \in \mathbb{R}^{d}, \quad k \in \Sigma
$$

formula (3.2) at fixed $k$ is considered as an equation for

$$
\psi=e^{i k x} \mu(x, k),
$$

where $\mu$ is sought in $L^{\infty}\left(\mathbb{R}^{d}\right)$; as a corollary of (3.2), $\psi$ satisfies $(1.1) ; h$ of (3.4) is a generalized "scattering" amplitude in the complex domain at zero energy.

Note that, actually, $G, \psi, h$ of (3.1)-(3.5) are zero energy restrictions of functions introduced by Faddeev as extentions to the complex domain of some functions of the classical scattering theory for the Schrödinger equation at positive energies. In addition, $G, \psi, h$ in their zero energy restriction were considered for the first time in [3]. The Faddeev functions $G, \psi, h$ were, actually, rediscovered in [3].

We recall also that, under the assumptions of Theorem 2.1

$$
\mu(x, k) \rightarrow 1 \text { as }|\operatorname{Im} k| \rightarrow \infty \text { (uniformly in } x \text { ) }
$$

and, for any $c>1$,

where $x \in \mathbb{R}^{d}, k \in \Sigma$;

$$
|\mu(x, k)|<c \text { for }|\operatorname{Im} k| \geq \rho_{1}(R, D, m, c),
$$

$$
|\hat{v}(p)-h(k, l)| \leq \frac{C_{3}(D, m) R^{2}}{\rho} \text { for }(k, l) \in \Theta, p=k-l,|\operatorname{Im} k|=|\operatorname{Im} l|=\rho \geq \rho_{2}(R, D, m),
$$


where

$$
\hat{v}(p)=\left(\frac{1}{2 \pi}\right)^{d} \int_{\mathbb{R}^{d}} e^{i p x} v(x) d x, \quad p \in \mathbb{R}^{d} .
$$

Results of the type (3.8), (3.9) go back to [3]. Results of the type (3.10), (3.11) (with less precise right-hand side in (3.11)) go back to [9]. Estimates (3.8), (3.11) are related also with some important $L_{2}$-estimate going back to [19] on the Green function $g$ of (3.1).

For more information on properties of the Faddeev functions $G, \psi, h$, see [9], citeNo2, [17] and references therein.

In the next section we recall that Problem 1.1 (of Introduction) admits a reduction to the following inverse "scattering" problem:

Problem 3.1. Given $h$ on $\Theta$, find $v$ on $\mathbb{R}^{d}$.

\section{Reduction of [14], [15]}

Let conditions (1.2), (1.3) hold for potentials $v_{1}$ and $v_{2}$, where $D$ is fixed. Let $\Phi_{i}, \psi_{i}, h_{i}$ denote the DtN map $\Phi$ and the Faddeev functions $\psi, h$ for $v=v_{i}, i=1,2$. Let also $\Phi_{i}(x, y)$ denote the Schwartz kernel $\Phi(x, y)$ of the integral operator $\Phi$ for $v=v_{i}, i=1,2$. Then (see [15] for details):

$$
h_{2}(k, l)-h_{1}(k, l)=\left(\frac{1}{2 \pi}\right)^{d} \int_{\partial D \partial D} \psi_{1}(x,-l)\left(\Phi_{2}-\Phi_{1}\right)(x, y) \psi_{2}(y, k) d y d x,
$$

where $(k, l) \in \Theta$

$$
\begin{gathered}
\psi_{2}(x, k)=\psi_{1}(x, k)+\int_{\partial D} A(x, y, k) \psi_{2}(y, k) d y, x \in \partial D, \\
A(x, y, k)=\int_{\partial D} R_{1}(x, z, k)\left(\Phi_{2}-\Phi_{1}\right)(z, y) d z, x, y \in \partial D, \\
R_{1}(x, y, k)=G(x-y, k)+\int_{\mathbb{R}^{d}} G(x-z, k) v_{1}(z) R_{1}(z, y, k) d z, x, y \in \mathbb{R}^{d},
\end{gathered}
$$

where $k \in \Sigma$. Note that: (4.1) is an explicit formula, (4.2a) is considered as an equation for finding $\psi_{2}$ on $\partial D$ from $\psi_{1}$ on $\partial D$ and $A$ on $\partial D \times \partial D$ for each fixed $k,(4.2 \mathrm{~b})$ is an explicit formula, (4.3) is an equation for finding $R_{1}$ from $G$ and $v_{1}$, where $G$ is the function of (3.1).

Note that formulas and equations (4.1)-(4.3) for $v_{1} \equiv 0$ were given in [14] (see also [9] (Note added in proof), [12], [13]). In this case $h_{1} \equiv 0, \psi_{1}=e^{i k x}, R_{1}=G(x-y, k)$. Formulas and equations (4.1)-(4.3) for the general case were given in [15].

Formulas and equations (4.1)-(4.3) with fixed background potential $v_{1}$ reduce Problem 1.1 (of Introduction) to Problem 3.1 (of Section 3).

\section{Reconstruction of [14] in the Born approximation}

In the Born approximation, that is in the linear approximation near zero potential, we have that

$$
\begin{gathered}
h(k, l) \approx \hat{v}(k-l), \\
h(k, l) \approx\left(\frac{1}{2 \pi}\right)^{d} \iint_{\partial D \partial} e^{-i l x}\left(\Phi-\Phi_{0}\right)(x, y) e^{i k y} d x d y,
\end{gathered}
$$

where $(k, l) \in \Theta, \hat{v}$ is defined by (3.12), $\Phi_{0}$ denotes the DtN map for $v \equiv 0$. 
Formulas (5.1), (5.2) follow from (3.1)-(3.4) and (4.1). Formulas (5.1), (5.2) imply, in particular, that

$$
\begin{aligned}
& \hat{v}(p) \approx\left(\frac{1}{2 \pi}\right)^{d} \iint_{\partial D \partial D} e^{-i l(p) x}\left(\Phi-\Phi_{0}\right)(x, y) e^{i k(p) y} d x d y, \\
& k(p)=\frac{p}{2}+i \frac{|p|}{2} \gamma(p), \quad l(p)=-\frac{p}{2}+i \frac{|p|}{2} \gamma(p), \quad p \in \mathbb{R}^{d},
\end{aligned}
$$

where $\gamma(p)$ is a piecewise continuous function of $p \in \mathbb{R}^{d}$ with values in $\mathbb{S}^{d-1}$ and such that

$$
\gamma(p) p=0, \quad p \in \mathbb{R}^{d} .
$$

One can see that formula (5.3) gives a reconstruction method for Problem 1.1, $d \geq 2$, in the Born approximation.

An approximate reconstruction method based on (5.1), (5.2) for Problem 1.1 in dimension $d \geq 2$ was proposed for the first time in [14].

In the next section we show that, in the Born approximation, Theorem 2.2 (of Section 2) follows, actually, from (5.3).

\section{Proof of Theorem 2.2 in the Born approximation}

We have that

$$
\begin{gathered}
v_{1}(x)-v_{2}(x)=\left(\int_{|p|<\rho}+\int_{|p|>\rho}\right) e^{-i p x}\left(\hat{v}_{1}(p)-\hat{v}_{2}(p)\right) d p, \\
\left|v_{1}(x)-v_{2}(x)\right| \leq I_{1}(\rho)+I_{2}(\rho), \\
I_{1}(\rho)=\int_{|p|<\rho}\left|\hat{v}_{1}(p)-\hat{v}_{2}(p)\right| d p, \\
I_{2}(\rho)=\int_{|p|>\rho}\left|\hat{v}_{1}(p)-\hat{v}_{2}(p)\right| d p,
\end{gathered}
$$

where $x \in \mathbb{R}^{d}, \rho>0$.

The assumptions $\left\|v_{j}\right\|_{m, 1} \leq R, j=1,2$, imply that

$$
\left|\hat{v}_{1}(p)-\hat{v}_{2}(p)\right| \leq \frac{C_{4}(d, m) R}{(1+|p|)^{m}}, \quad p \in \mathbb{R}^{d} .
$$

Using (5.3) we obtain that

$$
\left|\hat{v}_{1}(p)-\hat{v}_{2}(p)\right| \leq C_{5}(D) e^{L \rho}\left\|\Phi_{1}-\Phi_{2}\right\|, \quad|p| \leq \rho, C_{5}(D)=\left(\frac{1}{2 \pi}\right)^{d} \int_{\partial D} d x, \quad L=\max _{x \in D}|x|,
$$

where $\|\cdot\|$ is defined according to $(2.4)$.

Formulas (6.2b), (6.4) imply that

$$
I_{1}(\rho) \leq C_{6}(D) \rho^{d} e^{L \rho}\left\|\Phi_{1}-\Phi_{2}\right\| \leq C_{6}(D) e^{L_{1} \rho}\left\|\Phi_{1}-\Phi_{2}\right\|, \quad \rho>0, C_{6}(D)=C_{5}(D) \int_{\theta \in \mathbb{S}^{d-1}} d \theta, \quad L_{1}=L+d
$$

Formulas (6.2c), (6.3) imply that

$$
I_{2}(\rho) \leq C_{7}(d, m) R \rho^{-(m-d)}, \quad \rho>0 .
$$


Let

Then, due to $(6.5),(6.6)$,

$$
\alpha \in] 0,1\left[\text { be fixed, }\left\|\Phi_{1}-\Phi_{2}\right\|=\delta, \quad \rho=\lambda \ln \left(1+\delta^{-1}\right), \quad \lambda=\frac{1-\alpha}{L_{1}} .\right.
$$

$$
I_{1}(\rho) \leq C_{6} \delta\left(1+\delta^{-1}\right)^{\lambda L_{1}}=C_{6}(1+\delta)^{1-\alpha} \delta^{\alpha}, I_{2}(\rho) \leq C_{7} R\left(\lambda \ln \left(1+\delta^{-1}\right)\right)^{-(m-d)} .
$$

Estimate (2.8) for $\delta=\left\|\Phi_{1}-\Phi_{2}\right\| \leq 1 / 2$ follows from (6.2a), (6.8). Estimate (2.8) in the general case (with modified $C_{2}$ ) follows from (2.8) for $\delta \leq 1 / 2$ and the assumptions that $\left\|v_{j}\right\|_{L^{1}\left(\mathbb{R}^{d}\right)} \leq R, j=1,2$.

Thus, in the Born approximation Theorem 2.2 is proved. (This proof is valid also for $d=2$ ).

\section{REFERENCES}

[1] G. Alessandrini, Stable determination of conductivity by boundary measurements, Appl. Anal. 27 (1988), 153-172.

[2] H. Ammari, An Introduction to Mathematics of Emerging Biomedical Imaging, Math. \& Appl., Vol. 62, Springer, Berlin, 2008.

[3] R.Beals and R.R. Coifman, Multidimensional inverse scattering and nonlinear partial differential equations, Proc. Symp. Pure Math. 43 (1985), 45-70.

[4] R.M. Brown and G. Uhlmann, Uniqueness in the inverse conductivity problem for nonsmooth conductivities in two dimensions, Comm. Partial Diff. Eq. 22 (1997), 1009-1027.

[5] A.-P. Calderón, On an inverse boundary value problem, Seminar on Numerical Analysis and its Applications to Continuum Physics (Rio de Janeiro, 1980), pp.65-73, Soc. Brasil. Mat. Rio de Janeiro, 1980.

[6] L.D. Faddeev, Growing solutions of the Schrödinger equation, Dokl. Akad. Nauk SSSR 165 (1965), 514-517 (in Russian); English Transl.: Sov. Phys. Dokl. 10 (1966), 1033-1035.

[7] L.D. Faddeev, Inverse problem of quantum scattering theory II, Itogi Nauki i Tekhniki, Sovr. Prob. Math. 3 (1974), 93-180 (in Russian); English Transl.: J.Sov. Math. 5 (1976), 334-396.

[8] I.M. Gelfand, Some problems of functional analysis and algebra, Proceedings of the International Congress of Mathematicians, Amsterdam, 1954, pp.253-276.

[9] G.M. Henkin and R.G. Novikov, The $\bar{\partial}$ - equation in the multidimensional inverse scattering problem, Uspekhi Mat. Nauk 42(3) (1987), 93-152 (in Russian); English Transl.: Russ. Math. Surv. 42(3) (1987), 109-180.

[10] R. Kohn and M. Vogelius, Determining conductivity by boundary measurements II, Interior results, Comm. Pure Appl. Math. 38 (1985), 643-667.

[11] N. Mandache, Exponential instability in an inverse problem for the Schrödinger equation, Inverse Problems 17 (2001), 14351444.

[12] A.I. Nachman, Reconstructions from boundary measurements, Ann. Math. 128 (1988), 531-576.

[13] A.I. Nachman, Global uniqueness for a two-dimensional inverse boundary value problem, Ann, Math. 142 (1995), 71-96.

[14] R.G. Novikov, Multidimensional inverse spectral problem for the equation $-\Delta \psi+(v(x)-E u(x)) \psi=0$, Funkt. Anal. i Pril. 22(4) (1988), 11-22 (in Russian); English Transl.: Funct. Anal. and Appl. 22 (1988), 263-272.

[15] R.G. Novikov, Formulae and equations for finding scattering data from the Dirichlet-to-Neumann map with nonzero background potential, Inverse Problems 21 (2005), 257-270.

[16] R.G. Novikov, The $\bar{\partial}$ - approach to approximate inverse scattering at fixed energy in three dimensions, International Mathematics Research Papers, 2005:6, (2005), 287-349.

[17] R.G. Novikov, On non-overdetermined inverse scattering at zero energy in three dimensions, Ann. Scuola Norm. Sup. Pisa Cl. Sci. 5 (2006), 279-328.

[18] V.P. Palamodov, Gabor analysis of the continuum model for impedance tomography, Ark. Mat. 40 (2002), 169-187.

[19] J. Sylvester and G. Uhlmann, A global uniqueness theorem for an inverse boundary value problem, Ann. Math. 125 (1987), 153-169. 